\title{
Evaluación de la eficiencia de tres fertilizantes edáficos sobre el rendimiento y calidad del zapallo (Cucurbita maxima var. Unapal- Mandarino) Evaluation of the efficiency of three soil fertilizers on the yield and quality of zapallo (Cucurbita maxima var. Unapal - Mandarino)
}

\author{
Avaliação da eficiência de três fertilizantes do solo sobre a \\ produtividade e qualidade de abóbora (Cucurbita maxima \\ var. Unapal- Mandarino)
}

\author{
Juan Carlos Menjivar-Flores ${ }^{1}$, Cristian Felipe Enciso Murillo² \& Harol E. Martínez Cordoba ${ }^{3}$ \\ ${ }^{1}$ Ingeniero Agrónomo, Magister en suelos y aguas, Doctor en Ciencias del suelo. ${ }^{2}$ Estudiante de \\ Ingeniería Agronómica, Facultad de Ciencias Agropecuarias, Universidad Nacional de Colombia sede \\ Palmira. 3ingeniero Agrónomo, Magíster en Ciencias énfasis en Entomología. \\ 1, 2 Facultad de Ciencias Agropecuarias. Universidad Nacional de Colombia Sede Palmira. Colombia. \\ ${ }^{3}$ Investigación \& Desarrollo. Departamento Técnico. COSMOAGRO S.A. Palmira. Colombia
}

jcmenjivarf@unal.edu.co', cfencisom@unal.edu.co², hemartinezc@gmail.com³

\section{Resumen}

La investigación evaluó la eficiencia agronómica del cultivo de zapallo (Cucurbita máxima) var Unapal Mandarino por efecto de la aplicación de tres fertilizantes edáficos en el desarrollo, producción y rendimiento del cultivo en condiciones del Valle del Cauca, Colombia. Se estableció un diseño completamente al azar con tres tratamientos y cuatro repeticiones para un total de 12 unidades experimentales. Los tratamientos evaluados fueron T1: dosis comercial, T2: dosis comercial $+25 \%$. T3: dosis comercial $+50 \%$. La variables de respuesta evaluadas incluyen: peso total de frutos y número de frutos por unidad experimental, peso del fruto, diámetro polar, diámetro ecuatorial, índice de esfericidad, espesor de la pulpa, color de pared, número total de semillas, peso total de semillas, peso de 100 semillas, peso unitario de semilla y $\%$ materia seca. Los resultados mostraron que la eficiencia agronómica en los tratamientos dosis comercial y dosis comercial $+25 \%$ no presentaron diferencias significativas, sin embargo en el tratamiento con dosis comercial $+50 \%$ se encontraron diferencias significativas especialmente en variables de peso total de frutos, diámetro ecuatorial del fruto, diámetro polar del fruto, número total de semillas por fruto y en el peso total de semillas por fruto, por tanto al aumentar la dosis comercial del fertilizante, se aumentan las características agronómicas del fruto.

Palabras clave: eficiencia agronómica, fertilizante edáfico, producción, rendimiento.

\section{Abstract \\ The research assessed the agronomic efficiency of cultivation of zapallo (Cucurbita máxima) var Unapal Mandarin by effect of the application of three soil fertilizer in the development, production and crop}


yield in the Valle del Cauca, Colombia. A completely randomized design with three treatments and four replications for a total of 12 experimental units was established. The evaluated treatments were T1: commercial dose, T2: commercial dose $25 \%$. T3: commercial dose $50 \%$. The response variables evaluated included: total weight of fruits and number of fruits per experimental unit, weight of the fruit, polar diameter, equatorial diameter, index of sphericity, pulp thickness, color of wall, total number of seeds, total seed weight, weight of 100 seeds, unit weight of seed and percent of dry matter. The results showed that the agronomic efficiency in commercial dosage treatments and plus $25 \%$ commercial dose had no significant differences, however in the treatment with dose commercial $50 \%$ happen significant differences especially in variables of total weight of fruits, equatorial diameter of the fruit, the polar diameter of the fruit, total number of seeds per fruit and the total weight of seeds per fruit, therefore increasing the commercial fertilizer dose the agronomic characteristics of the fruit are increased.

Keys word: agronomic efficiency, soil fertilizer, production, yield

\section{Resumo}

A pesquisa avalio a eficiência agronômica da cultura de abobora variedade "Unapal
Mandarino" por efeito da aplicação de três fertilizantes edáficos no desenvolvimento, produção e produtividade da cultura em condições do Valle del Cauca, Colômbia. Foi estabelecido um delineamento experimental inteiramente casualizado com três tratamentos e quatro repetições para um total de 12 unidades experimentais. Os tratamentos avaliados foram T1: dose comercial, T2: dose comercial $+25 \%$, T3: dose comercial + $50 \%$. Os caracteres avaliados foram: peso total de frutos e número de frutos por parcela experimental, peso do fruto, diâmetro polar, diâmetro equatorial, índice de esfericidade, espessura da polpa, cor da parede, número total de sementes, peso total de sementes, peso de 100 sementes, peso unitário de semente e \% de matéria seca. Os resultados mostraram que a eficiência agronômica nos tratamentos dose comercial e dose comercial $+25 \%$ não apresentaram diferencias significativas especialmente nos caracteres peso total de frutos, diâmetro equatorial de fruto, dímetro polar de fruto, número total de semente por fruto e peso total de sementes por fruto. Por tanto, ao passo que aumenta a dose comercial do fertilizante, aumentam as características agronômicas do fruto.

Palavras-chave: eficiência agronômica, fertilizante edáficos produção, rendimento

\section{Introducción}

El género Cucurbita es importante por formar parte de la alimentación básica en muchas regiones de América, Asia y Europa (Vallejo \& Estrada, 2004). En 2015 se sembraron en el mundo 1.797 .195 ha $^{-1}$ de esta hortaliza, con una producción total de $24.679 .859 \mathrm{t}$ y un rendimiento de $137.324 \mathrm{~kg} \mathrm{ha}^{-1}$ (FAO, 2014). El zapallo en Colombia está comenzando a jugar un papel importante en la economía campesina, especialmente por el tamaño, forma y color de fruto, grosor y textura de pulpa, color y tamaño de la semilla, según Vallejo \& Estrada (2004), se establece en huertos caseros, en climas cálido y templado, entre 0 y $2000 \mathrm{msnm}$. En el Valle del Cauca, Colombia, en el 2014 se reportaron 228,8 ha, con una producción de 3,913 t y un rendimiento promedio de 17,10 t/ha-1 (Agronet, 2014), esto refleja la importancia de esta hortaliza en la agroindustria tanto a nivel nacional como mundial.

Los departamentos de mayor producción en Colombia son Cesar (15974 t), Caldas (9850 t), Boyacá (8428 t), seguido de Magdalena, Santander y La Guajira (Agronet, 2014). Donde es utilizado tanto para fines alimenticios como medicinales y agroindustriales. 
El Zapallo a nivel nutricional provee carbohidratos, B-caroteno (provitamina A), ácido ascórbico (vitamina C), minerales (calcio, hierro, fósforo) y aminoácidos como tiamina y niacina (Caicedo, 1993; Vallejo \& Mosquera, 1998; Carmo, 2009); se consume en forma directa (sopas, cremas, dulces, purés, jugos, pastelería y compotas) y de forma indirecta como materia prima para la agroindustria (harinas y deshidratados) (Espitia, 2004). Según Maynard et al., (2004) el fruto de zapallo contiene entre 4.4 y $14.5 \%$ de proteína cruda, una digestibilidad de la materia seca (MS) superior a $80 \%$.

El cultivar Unapal Mandarino, es una variedad con tolerancia a plagas y enfermedades comunes en las regiones similares a las del Valle del Cauca, Colombia, mostrando buena adaptación. Este cultivar se diferencia de otros porque tiene tallos gruesos, redondos, hojas anchas de bordes enteros y lisos, color verde claro, es una planta monoica con inicio de floración entre los 40-45 días para flores machos y los 45-55 días para flores hembras, los frutos son gruesos, redondo o aplanado de superficie lisa o ligeramente costilladas (Vallejo \& Estrada, 2004)

Los nutrientes en las plantas se convierten en los más importantes insumos para el incremento de los rendimientos, es así como el un buen manejo de la nutrición con el fin de incrementar las cantidades de nutrientes en los sistemas de producción, se ha convertido el principal desafío para la seguridad alimentaria y el desarrollo rural (FAO, 1999).

En cualquier cultivo es fundamental la fertilización, esta puede ser orgánica o química, en ambos casos lo importante es obtener buena productividad. Es así como en Colombia, es escasa la información sobre los requerimientos nutricionales de zapallo, ya que se considera un cultivo de poca importancia. Sin embargo éste responde positivamente al suministro de nutrientes adicionales al suelo, incrementando el peso de los frutos, y con ello mejorando los rendimientos y la productividad, Según Ribeiro (2008) la fertilización representa entre el $20 \%$ a $30 \%$ del costo total de producción.
El cultivo de zapallo, a diferencia de otras hortalizas, requiere una moderada cantidad de nitrógeno, pero si altas cantidades de potasio y fósforo (Sackett, 1975), el mismo autor plantea que la relación optima en entre nitrógeno, fosforo y potasio debe mantenerse en una proporción 2:1:3, en ese sentido cuando existe excesos de nitrógeno, el crecimiento vegetativo se vuelve exuberante pero ante un desbalance por carencia de fosforo, potasio o calcio, los frutos pueden deformarse o no alcanzar el tamaño adecuado. En zapallos híbridos se utilizan dosis de fertilización orgánica-mineral, poco evaluadas, pero se recomienda una aplicación de fertilización nitrogenada entre 80 y $100 \mathrm{~kg}$ ha-1 $^{-1}$ (Carmo, 2009)

Para los zapallos híbridos, estudios realizados por Santos et al., (2012) muestran que los nutrientes $\mathrm{N}, \mathrm{K}, \mathrm{S}$ y $\mathrm{Cu}$ se acumulan especialmente en los frutos, en cuanto el $\mathrm{P}, \mathrm{Ca}, \mathrm{Mg}, \mathrm{Zn}, \mathrm{Fe}$ y $\mathrm{Mn}$ en la parte vegetativa, por lo tanto se debe dar atención a las concentraciones de N, P y K por ser los nutrientes más exportados. En el contexto del Valle del Cauca el aporte de nutrientes por fertilizantes de síntesis en el cultivo de zapallo no está respaldado por estudios basados en los requerimientos nutricionales del cultivo, es así como los productores tradicionales fertilizan de acuerdo a las recomendaciones que brindan las casas comerciales.

Debido a la gran posibilidad de expansión del cultivo para fines productivos, es necesario validar dosis adecuadas de nutrientes y así lograr aumentar las cosechas. Por ende el objetivo de este trabajo fue evaluar la eficiencia en el aporte de nutrientes de tres fertilizantes edáficos a tres diferentes dosis en rendimiento y calidad del zapallo var. Unapal- Mandarino.

\section{Materiales y métodos}

La investigación se realizó en el Centro Experimental de la Universidad Nacional de Colombia sede Palmira- CEUNP, localizado en el municipio de Candelaria, Valle del Cauca, Colombia, a $3^{\circ} 24^{\prime}$ 
norte y $76^{\circ} 26^{\prime}$ oeste, a una altitud de $980 \mathrm{msnm}$, con temperatura media anual de $24^{\circ} \mathrm{C}$, precipitación media anual de $1.009 \mathrm{~mm}$ y humedad relativa del $69 \%$.

Para la preparación, adecuación y siembra del cultivo se realizó una labranza de tipo mecánica, comenzando con un arado de vertederas y posteriormente una labranza secundaria con rotovator para dejar las partículas del terreno más finas.

Se realizaron semilleros de la variedad Unapal Mandarino de zapallo, del programa de hortalizas de la Universidad Nacional de Colombia sede Palmira, posteriormente a los quince (15) días se pasaron a campo. La siembra se realizó con el método de tres bolillo utilizando una distancia entre plantas de $2 \mathrm{~m}$ y distancia entre surcos de $3 \mathrm{~m}$, se establecieron cuatro surcos con una longitud de $80 \mathrm{~m}$ de largo y $3 \mathrm{~m}$ de ancho cada uno y se dejó $1 \mathrm{~m}$ en cada extremo como borde de protección, para un total de área de experimentación de 1120 m².

El control de arvenses se llevó cabo de manera mecánica, química y manual. Cuando se presentó un desarrollo exuberante de los tallos secundarios se realizó el proceso de disposición de guías alrededor del tallo principal, con el fin de favorecer un crecimiento ordenado y mantener un área de cobertura definida.

Para el proceso de fertilización se tomaron los análisis de suelos de CEUNP los cuales fueron realizados por el Laboratorio de Química de suelos de la Universidad Nacional Sede Palmira, los requerimientos nutricionales del cultivo se tomaron de acuerdo a los planteados por Caicedo (1993) quien menciona que las cantidades de N, $\mathrm{P}, \mathrm{K}$ para un cultivo de zapallo, dependen de la fertilidad y del tipo de suelo.

El aporte de los nutrientes se realizó de acuerdo a las etapas fenológicas de la planta (vegetativa, reproductiva y maduración). En cada etapa fenológica se realizaron aplicaciones manuales de fertilización, las cuales consistían en realizar un agujero con un radio de $5 \mathrm{~cm}$ alrededor del tallo principal de cada planta, humedecerlo y agregar el fertilizante edáfico + compuesto (Triple 18), las cantidades aplicadas durante el proceso de fertilización del cultivo se presentan en la Tabla 1.

Tabla 1. Tratamientos y dosis de fertilizantes utilizados en la investigación.

\begin{tabular}{lcc}
\hline $\begin{array}{l}\text { Fertilizante } \\
\text { Edáfico }\end{array}$ & Tratamiento & $\begin{array}{c}\text { Componentes } \\
\left(\mathbf{k g} / 60 \mathbf{m}^{2}\right)\end{array}$ \\
\hline FE-1 & DC $-\mathrm{P}-\mathrm{K}$ \\
\hline & DC+25\% & 3,52 \\
& DC+50\% & 5,43 \\
FE-2 & DC & 6,31 \\
& DC+25\% & 7,58 \\
& DC+50\% & 9,1 \\
FE-3 & DC & 6,07 \\
& DC+25\% & 7,58 \\
\hline
\end{tabular}

DC: Dosis comercial

Se utilizó un diseño de bloques completamente al Azar con un arreglo factorial de $2 \times 3$, donde el primer factor correspondió al efecto de las dosis y el segundo correspondió al efecto de los fertilizantes. Los tratamientos evaluados fueron T1: Dosis Comercial, T2: Dosis Comercial $+25 \%$. T3: Dosis Comercial $+50 \%$, para cada uno de los fertilizantes edáficos evaluados de acuerdo a los componentes químicos N-P-K $\left(\mathrm{FE} 1^{\circledR}, \mathrm{FE} 2^{\circledR}, \mathrm{FE} 3^{\circledR}\right)$, cada tratamiento tuvo cuatro repeticiones de seis plantas.

\section{Variables evaluadas en campo.}

Se realizó la cosecha de los frutos de cada una de las unidades experimentales en sacos de fique, y se llevaron a una báscula industrial para obtener Peso total y número de frutos por unidad experimental (ptfru) en kg. 
Variables evaluadas en laboratorio.

Para las mediciones en laboratorio se escogieron por cada unidad experimental frutos con características homogéneas, se secaron durante 24 horas a una temperatura ambiente de $\pm 20^{\circ} \mathrm{C}$ para evitar descomposición temprana del fruto y se evaluó:

-Peso del fruto (pufru). Se pesaron los frutos escogidos por cada unidad experimental en una balanza electrónica y se obtuvo el peso (g).

-Diámetro polar $\varnothing$ (DEPFRU). Con la ayuda del flexómetro se midió desde la base hasta el ápice del fruto la distancia $(\mathrm{cm})$, la cual se toma como diámetro polar.

-Diámetro ecuatorial $\otimes(D E F R U)$ Se realizó el corte longitudinal al fruto de zapallo, dividiéndose en dos y posteriormente con la ayuda de un flexómetro se midió el diámetro $(\mathrm{cm})$.

-Índice de esfericidad (IESTFRU) =

Relación entre el diámetro polar del fruto y diámetro ecuatorial del fruto. Valores $<1$ indican que el fruto tiende a ser alargado; valores $>1$ indican que el fruto tiende a ser aplanado o achatado, y valores $=1$ sugieren que el fruto es de forma redonda o esférica.

-Espesor de la pulpa (ESPULP). Con el flexómetro se midió el grosor $(\mathrm{cm})$ de la pulpa en la zona del máximo diámetro trasversal, desde la epidermis hasta el borde interno de la pulpa del fruto.

Color de pared (COLPULP). Se tomó según apreciación visual y de acuerdo a los descriptores que presenta el ABANICO Roche, el cual en cada paleta representa la tonalidad del color de la pulpa por medio de clasificación numérica de 1-15.

-Número total de semillas (NTSEMFR). Se extrajeron las semillas de los frutos escogidos y se agregaron a un balde con agua, se pasó tres veces por un tamiz de $2 \mathrm{~mm}$ para que retirar el mucilago, después se pasaron a bolsas de mallas en donde se esparcían para su posterior secado y conteo.

-Peso total de semillas (PTSEMFR). Después del secado, se tomaron cada una de las bolsas de mallas que contenía las semillas de los frutos por unidad experimental y se llevaron a una balanza de precisión para conocer su peso $(\mathrm{g})$

-Peso de 100 semillas (PE100SEM). De acuerdo al peso Total de Semillas obtenido, se realizó una regla de tres en donde se promedió el peso total de 100 semillas.

- Peso unitario de semilla (PUSEM). Se toma como base el peso total de semillas y se hace la conversión para conocer el peso unitario de la semilla.

-Materia seca \% (MSEC). Para la medición de materia seca se utilizó el protocolo establecido por el laboratorio de semillas de la Universidad Nacional de Colombia sede Palmira y se calculó mediante la siguiente formula:

\section{Resultados y discusión}

\section{Efecto de las diferentes dosis en los caracteres agronómicos.}

En las diferentes dosis evaluadas en los tres fertilizantes edáficos se encontró que al aumentar un $50 \%$ la dosis comercial se presentan diferencias significativas especialmente en variables como Peso Total de Frutos por unidad experimental (PTFRU), Diámetro Ecuatorial del fruto (DEFRU), Diámetro Polar del fruto (DPFRU), Número Total de Semillas por Fruto (NTSEMFR) y en el Peso Total de Semillas por Fruto en (PTSEMFR) (Tabla 2).

También se presentaron diferencias significativas entre la dosis comercial más $25 \%$ y la dosis comercial en las variables de Diámetro Ecuatorial del Fruto, Diámetro Polar del Fruto, Número total de Semillas por Fruto y Peso Total de Semillas por fruto. 


\section{Efecto en Materia seca (MSEC)}

El género C. maxima se considera que no posee materia seca elevada, los rangos oscilan entre
4 - 7\%. En la presente investigación se mantuvieron entre 7 a $8 \%$ de materia seca como se muestra en la Tabla 2.

TABLA 2. Análisis de varianza de las dosis de fertilizantes aplicadas sobre las variables agronómicas de zapallo variedad unapal mandarino.

\begin{tabular}{|c|c|c|c|c|c|c|}
\hline DESCRIPTOR & $\begin{array}{l}\ddot{X} T 1 \text { (Dosis } \\
\text { comercial) }\end{array}$ & $\begin{array}{c}\text { ẌT2 (Dosis } \\
\text { comercial +25\%) }\end{array}$ & $\begin{array}{l}\text { ẌT3 (Do- } \\
\text { sis comer- } \\
\text { cial +50\%) }\end{array}$ & $\begin{array}{c}\text { MEDIA } \\
(\ddot{\mathbf{X}})\end{array}$ & DMS $(0,05)$ & CV (\%) \\
\hline PTFRU & $11,783(\mathrm{~B})$ & $12,433(\mathrm{~B})$ & $19,517(\mathrm{~A})$ & 14,5778 & 3,4197 & 28,00437 \\
\hline NTFRU & $6,833(A)$ & $8,167(A)$ & $9,0(A)$ & 8 & 2,277 & 33,97814 \\
\hline PUFRU & 2069,2 (B) & $2,043,9(B)$ & $2606,3(A)$ & 2239,819 & 523,91 & 27,92401 \\
\hline DEFRU & $18,1792(\mathrm{~A})(\mathrm{B})$ & $19,2583(\mathrm{~B})$ & $20,5250(A)$ & 19,32083 & 1,8293 & 11,30307 \\
\hline DPFRU & $15,4458(\mathrm{~A})$ & $14,0083(\mathrm{~B})$ & $15,5417(\mathrm{~A})$ & 14,99861 & 1,4295 & 11,37774 \\
\hline IESTFRU & $1,18965(B)$ & $1,38090(A)$ & $1,32421(\mathrm{~A})$ & 1,298256 & 0,1116 & 10,26513 \\
\hline ESPULP & $3,3708(A)$ & $3,4750(A)$ & $3,6917(A)$ & 3,5125 & 0,3687 & 12,53229 \\
\hline COLPULP & $3,4583(A)$ & $3,9167(A)$ & $3,7917(A)$ & 2,72222 & 1,2997 & 41,6843 \\
\hline NTSEMFR & $374,17(\mathrm{~A})(\mathrm{B})$ & $359,21(B)$ & $410,96(A)$ & 381,4444 & 43,36 & 13,57047 \\
\hline PTSEMFR & $65,511(\mathrm{~A})(\mathrm{B})$ & $62,452(B)$ & $74,145(A)$ & 67,36938 & 9,3715 & 16,60654 \\
\hline P100SEM & $17,803(\mathrm{~A})$ & $17,623(\mathrm{~A})$ & $18,186(\mathrm{~A})$ & 17,87044 & 2,2442 & 14,99224 \\
\hline PUSEM & $0,17803(A)$ & $0,17623(\mathrm{~A})$ & $0,19019(A)$ & 0,181483 & 0,029 & 19,04941 \\
\hline MSEC & $8,0547(A)$ & $7,9261(\mathrm{~A})$ & $7,7621(\mathrm{~A})$ & 7,914314 & 1,3753 & 20,74577 \\
\hline
\end{tabular}

Letras diferentes indican diferencias significativas según la prueba DUNCAN $(p<0,05)$.

\section{Efecto de los tres fertilizantes edáficos en los caracteres agronómicos.}

De los fertilizantes edáficos analizados, se puede observar que el producto $\mathrm{FE}-1^{\circledR}$ presenta diferencia significativa en la variable de Número Total de Semillas por Fruto (NTSEMFR). El producto $\mathrm{FE}-3^{\circledR}$ se destaca principalmente en el Espesor de la Pulpa del Fruto (ESPULP) presentando una diferencia mínima significativa con los otros fertilizantes (Tabla 3). 
TABLA 3. Efecto de los fertilizantes edáficos en las variables agronómicas del zapallo variedad unapal mandarino.

\begin{tabular}{lcccc}
\hline DESCRIPTOR & FE-1® & FE-2® & FE-3® & DMS (0,05) \\
\hline PTFRU & $15,692(\mathrm{~A})$ & $14,858(\mathrm{~A})$ & $13,183(\mathrm{~A})$ & 3,4197 \\
NTFRU & $8,750(\mathrm{~A})$ & $7,833(\mathrm{~A})$ & $7,417(\mathrm{~A})$ & 2,277 \\
PUFRU & $2387,8(\mathrm{~A})$ & $2265,1(\mathrm{~A})$ & $2066,5(\mathrm{~A})$ & 523,91 \\
DEFRU & $19,6583(\mathrm{~A})$ & $19,4750(\mathrm{~A})$ & $18,8292(\mathrm{~A})$ & 1,0293 \\
DPFRU & $15,0042(\mathrm{~A})$ & $14,7792(\mathrm{~A})$ & $15,2125(\mathrm{~A})$ & 1,4295 \\
IESTFRU & $1,32217(\mathrm{~A})$ & $1,32506(\mathrm{~A})$ & $1,24754(\mathrm{~A})$ & 0,1116 \\
\hline ESPULP & $3,2875(\mathrm{~B})$ & $3,5625(\mathrm{~A})(\mathrm{B})$ & $3,6875(\mathrm{~A})$ & 0,3687 \\
COLPULP & $4,0833(\mathrm{~A})$ & $3,5417(\mathrm{~A})$ & $3,5417(\mathrm{~A})$ & 1,2997 \\
NTSEMFR & $409,08(\mathrm{~A})$ & $353,0(\mathrm{~B})$ & $382,25(\mathrm{~A})(\mathrm{B})$ & 43,36 \\
\hline PTSEMFR & $71,253(\mathrm{~A})$ & $64,562(\mathrm{~A})$ & $66,293(\mathrm{~A})$ & 9,3715 \\
\hline P100SEM & $17,711(\mathrm{~A})$ & $18,632(\mathrm{~A})$ & $17,269(\mathrm{~A})$ & 2,2442 \\
PUSEM & $0,18544(\mathrm{~A})$ & $0,18632(\mathrm{~A})$ & $0,17269(\mathrm{~A})$ & 0,029 \\
\hline MSEC & $7,9435(\mathrm{~A})$ & $7,7565(\mathrm{~A})$ & $8,0429(\mathrm{~A})$ & 1,3753 \\
\hline
\end{tabular}

Letras diferentes indican diferencias significativas según la prueba DUNCAN $(p<0,05)$.

\section{Peso total de frutos por unidad experimental (PTFRU)}

El analizar el peso total de frutos por unidad experimental permite tener una idea de la importancia de los fertilizantes para la producción comercial. Según el análisis de varianza y la prueba Duncan se observa que la Dosis Comercial fue la de menor rendimiento con $11,783 \mathrm{~kg} /$ parcela, seguida de la Dosis Comercial $+25 \%$ con $12,433 \mathrm{~kg} /$ parcela, no mostrando diferencias significativas entre ellas, mientras tanto al aumentar la Dosis Comercial un $50 \%$ se encuentran $19,517 \mathrm{~kg} /$ parcela, lo cual hace que se diferencie de los otros dos tratamientos.
En el caso de los fertilizantes a pesar que el análisis de varianza no muestra diferencias significativas, si se destaca el FE-1 con una media general de $15,692 \mathrm{~kg} /$ parcela, como el fertilizante edáfico de mejor efecto (Figura 1).

Estos resultados permiten suponer que es necesario aumentar la dosis un 50\% en cada uno de los tres fertilizantes edáficos para aumentar rendimientos. 


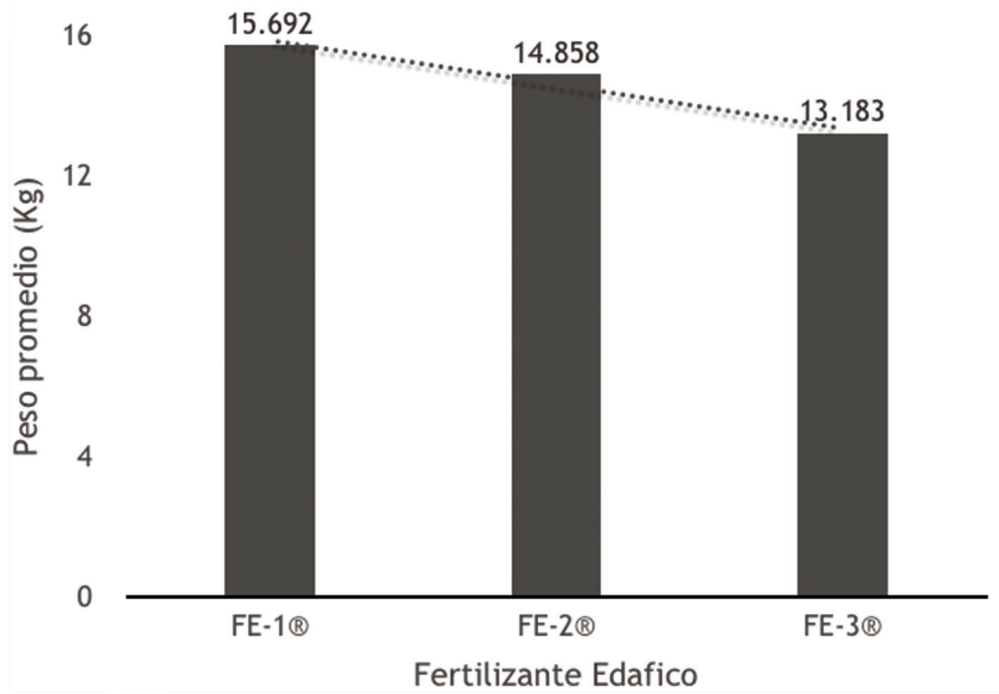

Figura 1. Efecto de los fertilizantes para la variable peso del fruto por unidad experimental.

\section{Número total de frutos (NTFRU)}

La Dosis Comercial $+50 \%$ presentó una media de frutos por parcela de 9,0 (1,5 frutos por las 6 plantas que había por unidad experimental) (Figura 2), pero no se muestra una diferencia significativa entre las dosis ni entre los fertilizantes, lo cual sugiere que el número total de frutos no está influenciada ni por las diferentes dosis ni el fertilizante sino más bien es un factor de tipo genético, en ese sentido y de acuerdo con Amariles y López (1994), se espera que un alto número de frutos conduzca a una mayor producción.

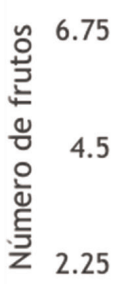

0
6.833

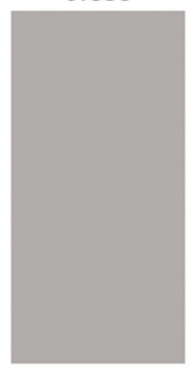

8.167
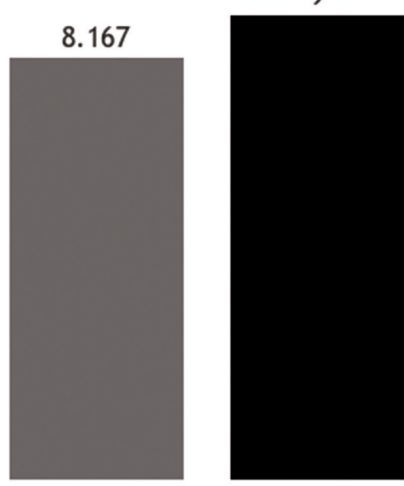

Promedio por Dosis evaludas

$\ddot{X} \mathrm{~T} 1$ (Dosis comercial)

$\ddot{X}$ T2 (Dosis comercial $+25 \%$ )

X T3 (Dosis comercial +50\%)

Figura 2. Promedio de número de frutos por unidad experimental según la dosis. 


\section{Peso Promedio Unitario del Fruto (PUFRU)}

Esta variable es de mucha importancia ya que se buscan zapallos con peso promedio en el mercado de 3 a $5 \mathrm{~kg}$. El análisis de varianza muestra que existen diferencias significativas en las diferentes dosis, mostrando que el tratamiento tres (Dosis comercial $+50 \%$ ) presentó los frutos más grandes, pero no superaron el peso promedio de $C$. maxima de $3,42 \mathrm{~kg}$ según lo mostrado por Amariles \& López, (1994) (Figura 3). Este resultado está relacionado con el peso total de frutos por unidad experimental y puede beneficiar al productor.

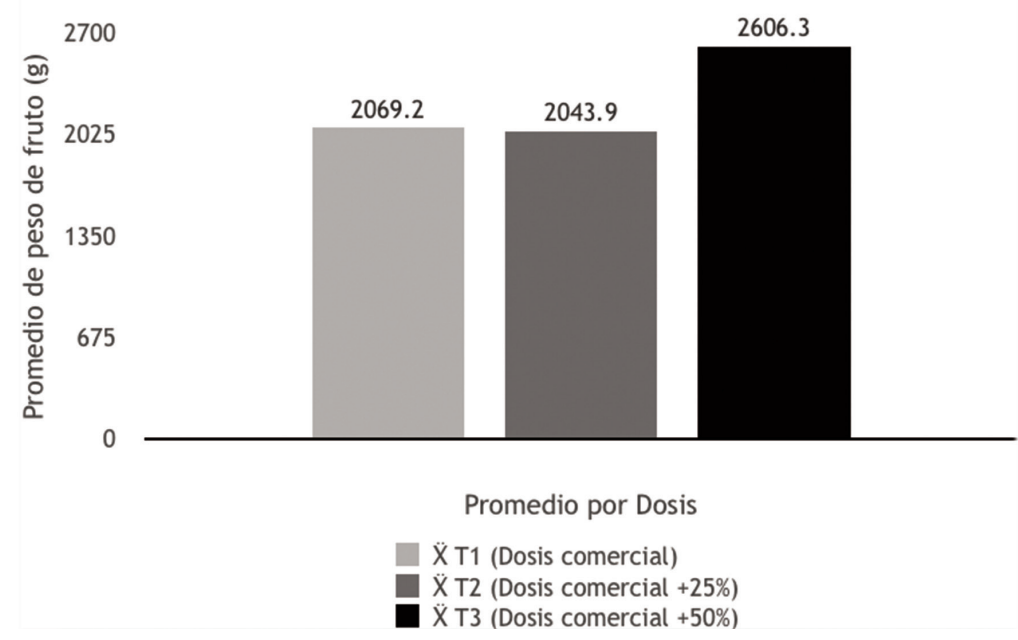

Figura 3. Peso promedio del fruto según las dosis evaluadas.

Diámetro ecuatorial (DEFRU), diámetro polar (DPFRU) e índice de esfericidad

Estas tres variables mostraron diferencias significativas entre las diferentes dosis evaluadas, encontrándose en el Diámetro Ecuatorial una diferencia mínima significativa (dms) de 1,8293 cm, destacándose el tratamiento tres (Dosis comercial $+50 \%$ ) con una media de 20,5250 cm; En la variable del diámetro polar se encontró diferencias significativas entre La Dosis Comercial y La Dosis Comercial $+50 \%$ con La Dosis Comercial $+25 \%$ (Figura 4 ).

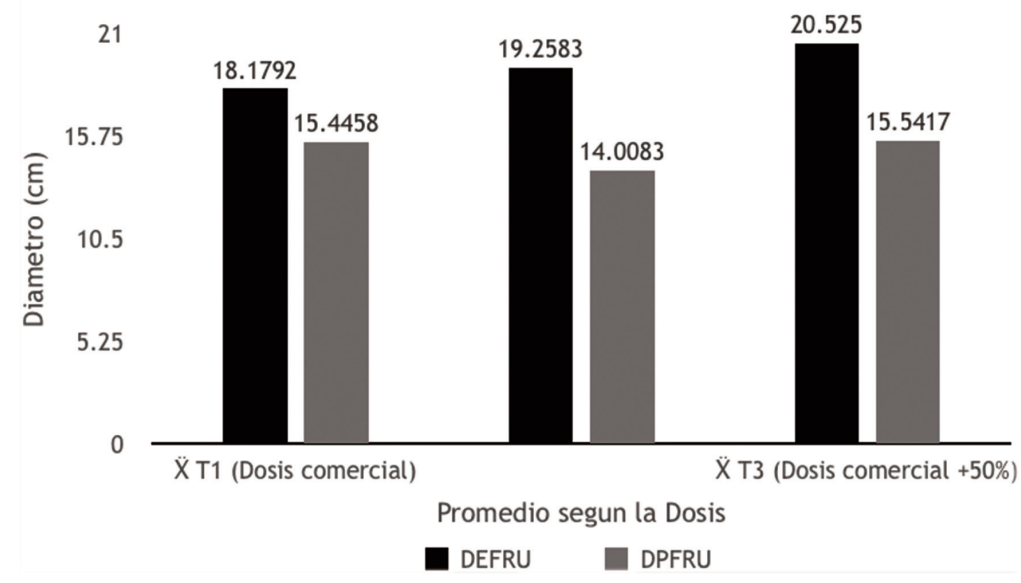

Figura 4. Relación entre el diámetro polar y ecuatorial de los frutos por tratamiento. 
El índice de esfericidad permite conocer el formato de fruto según las medidas de diámetro ecuatorial y polar. Actualmente existe la tendencia en el consumidor a preferir frutos de forma globular o redonda.

El género Cucurbita presenta una alta variabilidad para el formato del fruto, en esta investigación no se presentaron diferencias significativas entre las dosis ni los fertilizantes evaluados, los frutos en este ensayo tienden a ser aplanados y elípticos, ya que en las diferentes dosis evaluadas presentan medidas mayores a 1 (Figura 5),sin embargo a medida que se le aumenta la dosis el fruto tiende a ser más aplanado, lo cual va a facilitar el acondicionamiento, embalaje y comercialización, por parte del productor.

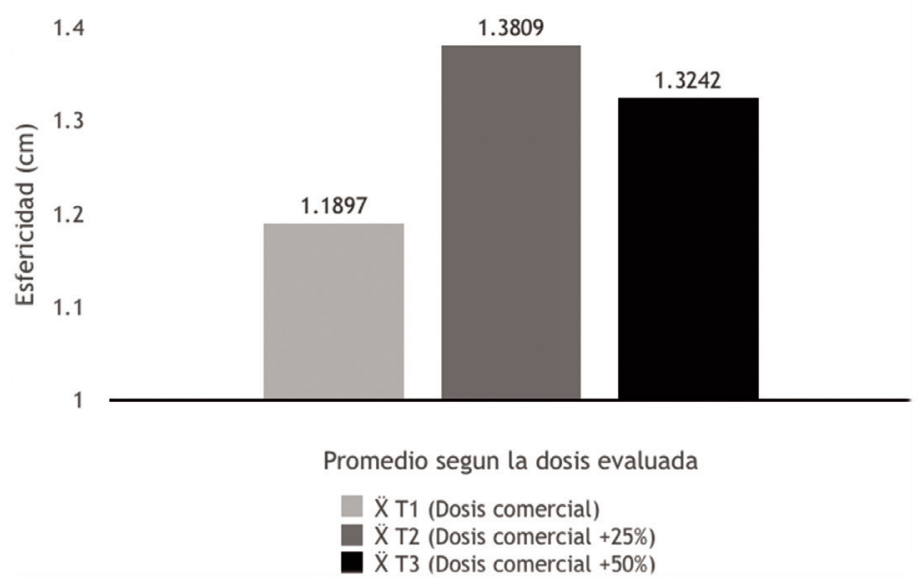

Figura 5. Relación del índice de esfericidad en los diferentes tratamientos evaluados.

Espesor de la pulpa. Esta variable es importante porque afecta la aceptación del consumidor, debido a que a mayor grosor parece más atractivo el fruto. El zapallo Unalpal-Mandarino es una variedad con menor espesor en comparación con otras variedades. En los ensayos no se observó una diferencia significativa entre los tres tratamientos, aunque hay una tendencia entre el aumento de la dosis con el aumento del espesor de la pulpa. Por su parte en el caso de los fertilizantes edáficos si se evidenciaron diferencias marcadas, siendo el mejor FE- $3^{\circledR}$, seguido de FE- $2^{\circledR}$ y por último FE- $1^{\circledR}$ (Figura 6) resultados similares reportan Tobar et al., (2009) quienes al evaluar diferentes familias de zapallo encontraron que en el carácter del espesor de pulpa (EP) los resultados variaban entre 3.34 (F11a) y $4.21 \mathrm{~cm}$ (F11b) con un promedio de $3.69 \mathrm{~cm}$ según la familia evaluada.

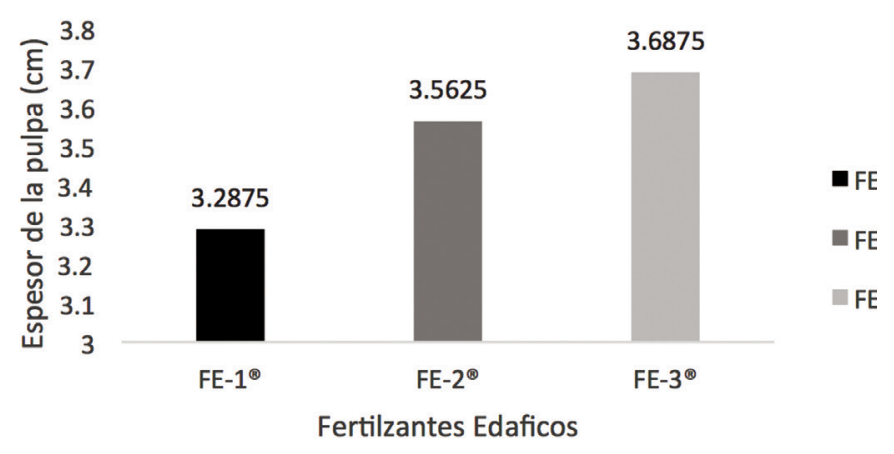

Figura 6. Espesor de la pulpa según los fertilizantes edáficos utilizados. 
Color de la pulpa (COLPULP). La calidad y el valor nutritivo del fruto hace referencia al color de la pulpa, encontrándose en éste principalmente vitamina A y minerales; el consumidor prefiere colores naranjas o amarillos. La variedad Unapal- Mandarino presenta colores claros respecto a otras variedades como Bolo verde, Llanogrande o Abanico 73. En esta investigación no se evidenciaron diferencias significativas para esta variable entre las dosis ni los fertilizantes aplicados. El color estuvo entre 3 y 4 específicamente, siempre siendo uniforme en todos los tratamientos. La característica del color de la pulpa está determinada por condiciones genéticas y bajo las condiciones de este ensayo, no se encontraron respuestas a coloración debido a niveles de nutrición.
Número total de semillas por fruto (NTSEMFR). En general, se acepta que la especie del género Cucurbita, tiene un alto número de semillas por frutos y la producción de semillas es un proceso relativamente sencillo, pues con un área pequeña se puede conseguir un adecuado suministro de semillas. El análisis de varianza mostró diferencias significativas entre las dosis, observándose un aumento de semillas al incrementar la Dosis Comercial $+50 \%$, esta última con una media de 410,96 semillas por fruto (Figura 7). Este resultado supera los valores reportado por Pérez M., (1997) evaluando diferentes genotipo, en los cuales el rango de semillas por fruto está de 284,6 en la Población. 34 a 340,8 en la Población 11.

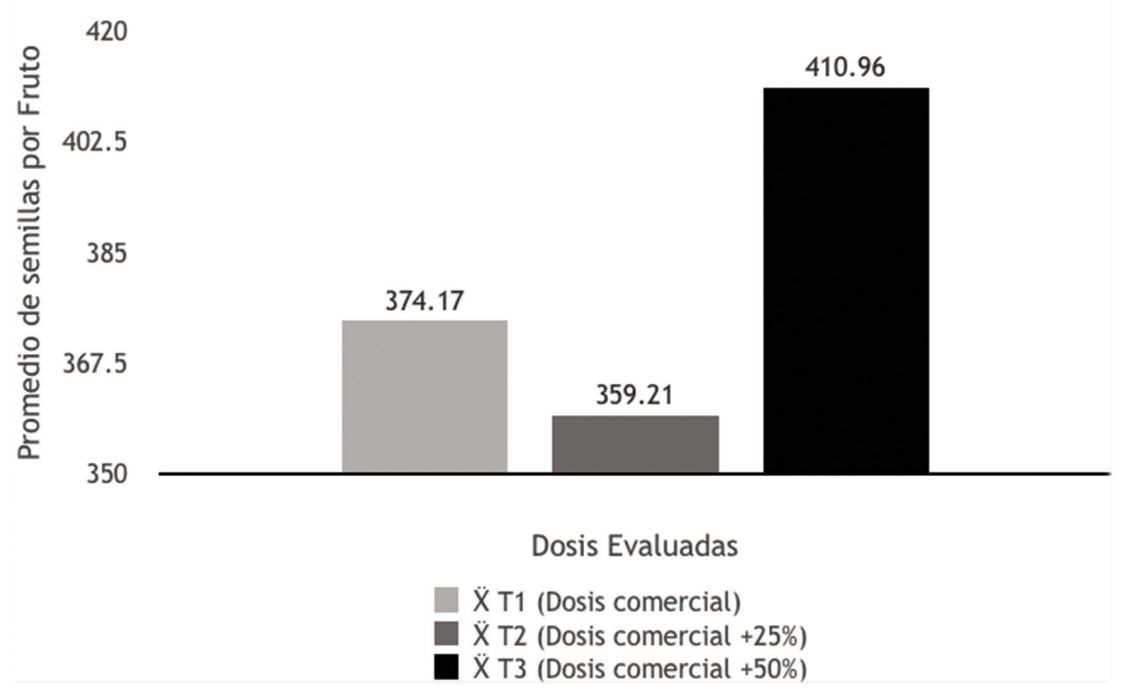

Figura 7. Número total de semillas por fruto según la dosis evaluada.

Peso total de semillas por fruto (PTSEMFR). Según los resultados obtenidos en esta variable se presentan diferencias significativas entre las dosis utilizadas, cuando se incrementó la dosis comercial un $50 \%$, aumento el peso a un promedio de 74,145 g. Este resultado está correlacionado con la variable nombrada anteriormente (Número total de semillas por fruto), lo que da a entender que al haber un aumento en el número de semillas, se aumenta el peso total de semillas por fruto (Figura 8). 


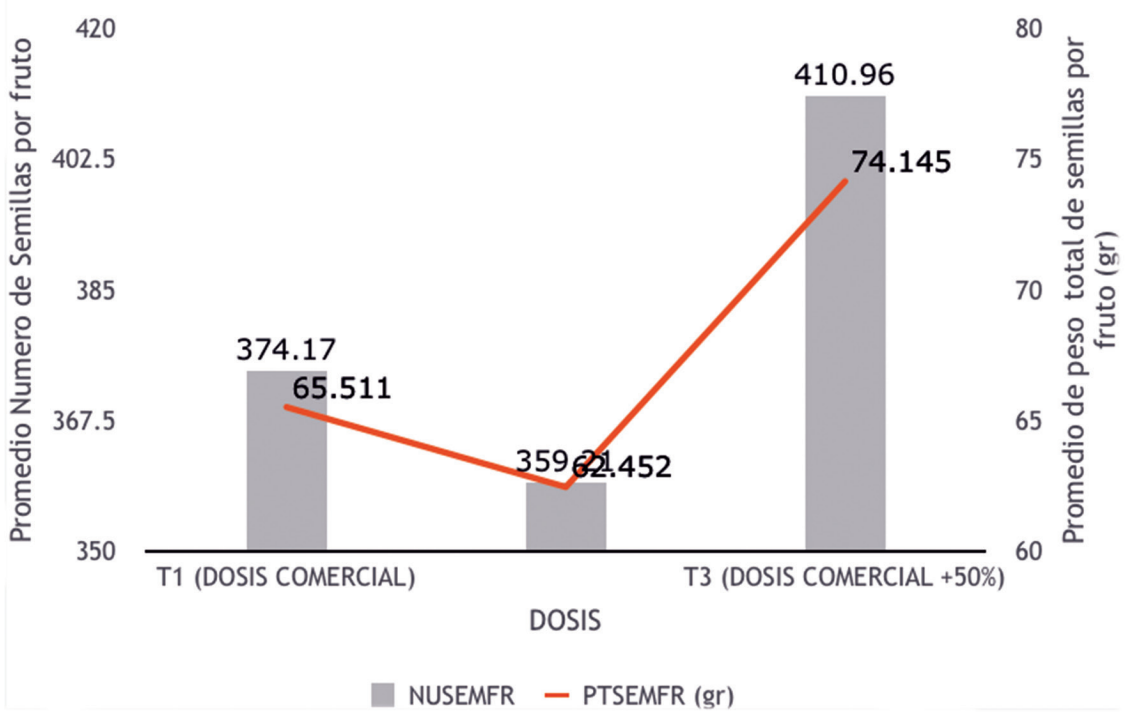

Figura 8. Correlación entre el número de semillas por fruto con peso total de semillas por fruto.

\section{Peso de 100 semillas (P100SEM) y peso unita- rio de semilla (PUSEM).}

El peso de 100 semillas es un factor relacionado con la calidad fisiológica de la semilla. En general, el peso de la semilla es mayor en C. maxima que en C. moschata, como lo señalan Amariles \& López, (1994). En las pruebas realizados en el laboratorio no se encontraron diferencias significativas en las dosis ni en los fertilizantes evaluados, el promedio de cada una de las dosis evaluadas de acuerdo a la variable de peso de 100 semillas fue para T1 Dosis Comercial 17,803; T2 Dosis Comercial $+25 \%$ 17,623; T3 Dosis Comercial $+50 \%$ 18,186 . En el caso de los fertilizantes se encontraron medias de $17,711 \mathrm{~g}$ para FE- $3^{\oplus}, 18,632 \mathrm{~g}$ para $\mathrm{FE}-2^{\circledR}$ y 17,269 g para $\mathrm{FE}-1^{\circledR}$, comparados con Pérez (1997) con valores de $13,77 \mathrm{~g}$, se obtuvieron semillas de mayor peso, corroborando que el efecto de la fertilización juega un papel importante en el peso de la semilla.

Para la variable Peso Unitario de Semillas, no se encontraron diferencias significativas entre la do- sis ni entre los fertilizantes, estos valores están correlacionados con los del peso de 100 semillas.

\section{Conclusiones}

Al aumentar la dosis comercial un $50 \%$ se favorece el aumento de los caracteres agronómicos en el cultivo, como el peso total de frutos, peso unitario de fruto, diámetro ecuatorial del fruto, diámetro polar del fruto, índice de esfericidad, número total de semillas por fruto y peso total de semillas por fruto, son los más relevantes frente a las dosis comercial y dosis comercial $+25 \%$; lo cual beneficiará al agricultor en el momento de venta de su producción.

Los fertilizantes influyeron en el comportamiento de variables de caracteres agronómicas de interés para el consumidor como son: Espesor de la Pulpa en el cual se destaca FE- $3^{\circledast}$ y Número Total de Semillas por Fruto en donde se recalcan $\mathrm{FE}-1^{\oplus}$ y FE-3 $3^{\circledR}$. 


\section{Literatura citada}

1. Amariles C \& López A. (1994). Aumento, caracterización, evaluación y selección de poblaciones promisorias de zapallo. (Tesis de pregrado) Universidad Nacional de Colombia sede Palmira. Valle del Cauca, Colombia.

2. Agronet. 2014. Cifras Agropecuarias.

3. Caicedo, L. A. (1993). Horticultura. Universidad Nacional de Colombia. Palmira. 6 (Ed.). (539 p). Manuscrito.

4. Carmo, G.A. (2009). Crescimento, nutrição e produção de cucurbitáceas cultivadas sob diferentes níveis de salinidade da água de irrigação e doses de adubação nitrogenada. (Tese Doutorado em Agronomia) Universidade Federal Rural do Semiárido. Recuperado de: http://www2.ufersa.edu.br/portal/view/uploads/setores/82/teses_2009/TESE_GILCIMAR_CARMO.pdf

5. Espitia, C. M. (2004). Estimación y análisis de parámetros genéticos en cruzamientos dialélicos de zapallo (Cucurbita moschata duch. ex poir). (Tesis de Doctorado) Escuela de Posgrados Universidad Nacional de Colombia sede Palmira. 206 p.

6. FAO. 2014. Estadísticas agrícolas mundiales. Recuperado de: http://www.fao.org/statistics/es/

7. FAO.1999. Guía para el manejo eficiente de la nutrición de las plantas. Recuperado de: ftp://ftp.fao.org/agl/agll/ docs/gepnms.pdf

8. Maynard, D. N; Elmostrom, G. W; Talcott, S. T; y Carle, R. B. (2004). El dorado and la estrella compact planta tropical pumpkin hyrids. Recuperado de http://www. gcrec.ifas.ufl.edu/tpmanuscript.htm

9. Pérez, S. (1997). Evaluación del rendimiento y calidad del fruto de tres poblaciones promisorias de zapallo Cucúrbita sp. (Tesis de pregrado) Ingeniero Agrónomo, Universidad Nacional de Colombia, Palmira. Valle del Cauca, Colombia
10. Ribeiro, D. S. (2008). Parâmetros agrometeorológicos de ambiente protegido com o cultivo de abóbora italiana sob adubação orgânica. (Tesis de Doctorado) Universidade Federal de Pelotas, Pelotas, Brasil. Recuperado de: http://www.ufpel.edu.br/tede/tde_arquivos/7/ TDE-2008-06-18T123717Z-269/Publico/Tese_\%20 Dagnon_\%20Ribeiro.pdf

11. Sackett, C. (1975). Squash. Fruit vegetable facts pointers. Washington. 2-9 p.

12. Santos, M. R; Aparecida, M; Moreira, M; Aparecida, C; Vidigal, S. M. (2012). Rendimento, qualidade e absorção de nutrientes pelos frutos de abóbora em função de doses de biofertilizante. Revista Horticultura Brasileira. 30 (01)

13. Tobar T, D.E., Vallejo C, F.A., Baena G, D. (2009). Evaluación de familias de zapallo (Cucurbita moschata Duch.) seleccionadas por mayor contenido de materia seca en el fruto y otras características agronómicas. Revista Acta Agronómica. 59 (1): 65-72

14. Vallejo, F. A \& Estrada, E. (2004).Producción de hortalizas de clima cálido. Universidad Nacional de Colombia sede Palmira.

15. Vallejo, F. A. \& Mosquera, S. E. (1998). Transferencia del gen $B u$ a poblaciones de zapallo, Cucurbita sp. Con crecimiento postrado. Acta Agronomica (48):7 -18.

\section{Conflicto de Intereses}

Los autores declaran no tener ningún conflicto de intereses 
